Original Research Paper

\title{
Unique Representation of Positive Integers as a Sum of Distinct Tribonacci Numbers
}

\author{
Salim Badidja and Abdelmadjid Boudaoud \\ Laboratory of Pure and Applied Mathematics (L.M.P.A.), \\ Faculty of Mathematics and Computer Sciences, University of Mohamed BOUDIAF of M'sila, M'sila, Algeria
}

Article history

Received: 28-12-2016

Revised: $16-02-2017$

Accepted: 15-03-2017

Corresponding Author:

Salim Badidja

Laboratory of Pure and Applied

Mathematics (L.M.P.A.),

Faculty of Mathematics and

Computer Sciences, University

of Mohamed BOUDIAF of

M'sila, M'sila, Algeria

Email: bsalimmah@gmail.com

\section{Introduction}

The tribonacci numbers $\left\{T_{n}\right\}_{1}^{\infty}$, are defined by:

$$
T_{n}=T_{n-1}+T_{n-2}+T_{n-3}, n \geq 4
$$

where, $T_{1}=T_{2}=1, T_{3}=2$ (Koshy, 2001). The first tribonacci numbers are $1,1,2,4,7,13,24, \ldots$.

Recall that in 1969 J. L Brown represents the integers by a sum of distinct Lucas numbers (Brown, Jr, 1969), also in 1986 Jukka Puhko treated the Fibonacci and Lucas representation and theorem of Lekkerkerker (Pihko, 1986), in the mathematical literature there are several references concerning the representation of a positive integer by a finite sum of elements of an infinite sequence of integers. Our main goal is to prove that every positive integer $N>0$ can be represented by $\sum_{1}^{\infty} \alpha_{m} T_{m}$, where $\alpha_{m}(m \geq 1)$ are either 1 or 0 . This representation is unique if the coefficients $\alpha_{m}(m \geq 1)$ satisfy:

$$
\left\{\begin{array}{l}
\alpha_{m} \alpha_{m+1}=0 \\
\alpha_{m} \alpha_{m+2}=0,
\end{array} \quad \text { for } m \geq 1\right.
$$

where, taking into account $T_{1}=T_{2}=1$, the uniqueness is in the following sense.

\section{Definition 1.1}

Two representations $\sum_{m=1}^{\infty} \alpha_{m} T_{m}$ and $\sum_{m=1}^{\infty} \beta_{m} T_{m}$ are called identical if:

- $\quad \alpha_{m}=\beta_{m}$ for $m \geq 1$ or

- $\quad \alpha_{m}=\beta_{m}$ for $m \geq 3$ and $\left(\alpha_{1}, \beta_{1}\right)=(1,0)$ and $\left(\alpha_{2}, \beta_{2}\right)=$ $(0,1)$ or $\left(\alpha_{1}, \beta_{1}\right)=(0,1)$ and $\left(\alpha_{2}, \beta_{2}\right)=(1,0)$

\section{Main Results}

\section{Theorem 2.1}

For every integer $N>0$, there exists an integer $i_{1}>0$ such that $N=\sum_{i=1}^{i_{1}} \alpha_{i} T_{i}$, where $\alpha_{i} \in\{0,1\}$.

\section{Proof}

Let $N>0$ be an integer. Firstly, we show that the sequence $\left(T_{i}\right)_{i \geq 1}$ satisfies:

$$
T_{i+1} \leq 2 T_{i}, \forall i \geq 1
$$

Indeed, we easily check this for $i=1,2,3$. For $i \geq 4$, we have by definition:

$$
T_{i+1}=T_{i}+T_{i-1}+T_{i-2} \leq T_{i}+\left(T_{i-1}+T_{i-2}+T_{i-3}\right)=2 T_{i}
$$



that:

Now, putting $\Delta_{0}=N$. Let $i_{1}$ be the largest integer such

$$
T_{i_{1}} \leq \Delta_{0}<T_{i_{1}+1}
$$

Putting $\Delta_{1}=\Delta_{0}-T_{i_{1}}$. If $\Delta_{1}=0$, then $\Delta_{0}=T_{i_{1}}$ i.e., $N=T_{i_{1}}$ and this completes the proof. Otherwise, $\Delta_{1}>0$ and in this case necessarily $\Delta_{1}=\Delta_{0}-T_{i_{1}}<T_{i_{1}}$ because if not then $\Delta_{0}-T_{i_{1}} \geq T_{i_{1}}$ yields $\Delta_{0} \geq 2 T_{i_{1}} \geq T_{i_{1}+1}$ but this contradicts the choice of $i_{1}$ which yields $T_{i_{1}} \leq \Delta_{0}<T_{i_{1}+1}$. Now we choose $i_{2}<i_{1}$ such that:

$$
T_{i_{2}} \leq \Delta_{1}<T_{i_{2}+1}
$$

Putting $\Delta_{2}=\Delta_{1}-T_{i_{2}}$. If $\Delta_{2}=0$, then $\Delta_{1}-T_{i_{2}}=0$, or $\Delta_{1}=T_{i_{2}}$ and consequently $\Delta_{1}=T_{i_{2}}=N-T_{i_{1}}$ since $\Delta_{0}=N$. Hence, $N=T_{i_{1}}+T_{i_{2}}$ and this ends the proof. Otherwise, $\Delta_{2}$ $>0$ and in this case necessarily $\Delta_{2}=\Delta_{1}-T_{i_{2}}<T_{i_{2}}$ if not then $\Delta_{1}-T_{i_{2}} \geq T_{i_{2}}$ which signifies that $\Delta_{1} \geq 2 T_{i_{2}} \geq T_{i_{2}+1}$ but this contradicts the fact that $T_{i_{2}} \leq \Delta_{1}<T_{i_{2}+1}$.

Continuing in this process, we obtain a decreasing sequence $i_{1}>i_{2}>i_{3}>\ldots>\ldots$ which should stabilize at some $i_{k}$. Hence $N=T_{i_{1}}+T_{i_{2}}+\ldots+T_{i_{k}}$ and consequently $N=\sum_{i=1}^{i_{1}} \alpha_{i} T_{i}$, where $\alpha_{i} \in\{0,1\}$ for $\left(1 \leq i \leq i_{1}\right)$.

\section{Proposition 2.1}

For every integer $n \geq 5$, we have:

$$
T_{n}=3+T_{n-2}+2 \sum_{i=2}^{n-3} T_{i}
$$

\section{Proof}

From the definition of $\left(T_{n}\right)_{n \geq 1}$, easily we have:

$$
T_{n}=T_{1}+T_{3}+T_{n-2}+2 \sum_{i=2}^{n-3} T_{i}, n \geq 5
$$

Or:

$$
T_{n}=3+T_{n-2}+2 \sum_{i=2}^{n-3} T_{i}, n \geq 5
$$

\section{Theorem 2.2}

Let $N \geq 1$ be an integer represented by $\sum_{m=1}^{\infty} \alpha_{m} T_{m}$, for $m$ $\geq 1$ :

$$
\alpha_{m} \in\{0,1\} \text { and }\left\{\begin{array}{l}
\alpha_{m} \alpha_{m+1}=0 \\
\alpha_{m} \alpha_{m+2}=0
\end{array}\right. \text {. }
$$

Then this representation is unique.

\section{Proof}

Before the proof we need the following lemma.

\section{Lemma 2.1}

Under the same assumption of theorem 2.2 and for $k$ $\geq 6$, we have:

$$
\sum_{m=1}^{k} \alpha_{m} T_{m}<2 \sum_{m=2}^{k-2} T_{m}+T_{k-2}
$$

\section{Proof}

Firstly we prove by induction that:

$$
2 T_{k-2}-T_{k-1}-1 \geq 0, k \geq 6
$$

This formula is satisfied for $k=6$, because:

$$
\begin{aligned}
2 T_{4}-T_{5}-1 & =8-7-1 \\
& =0 \geq 0
\end{aligned}
$$

Suppose that:

$$
2 T_{k-2}-T_{k-1}-1 \geq 0, k \geq 6
$$

and prove that:

$$
2 T_{(k+1)-2}-T_{(k+1)-1}-1=2 T_{k-1}-T_{k}-1 \geq 0
$$

In fact:

$$
\begin{aligned}
& 2 T_{k-1}-T_{k}-1=T_{k-1}-T_{k-2}-T_{k-3}-1 \\
& \left(T_{k-2}+T_{k-3}+T_{k-4}\right)-T_{k-2}-T_{k-3}-1 \\
& =T_{k-4}-1
\end{aligned}
$$

Since $T_{2}-1=0 \geq 0$ and the fact that $\left(T_{i}\right)_{i \geq 4}$ is strictly increasing, we have always:

$$
2 T_{k-1}-T_{k}-1 \geq 0, k \geq 6
$$

This completes the proof.

Returning to the proof of the lemma. Putting:

$$
\begin{aligned}
& L=2 \sum_{m=2}^{k-2} T_{m}-\sum_{m=1}^{k} \alpha_{m} T_{m}+T_{k-2} \\
& =\sum_{m=2}^{k-2}\left(2-\alpha_{m}\right) T_{m}-\alpha_{1}-\alpha_{k-1} T_{k-1}-\alpha_{k} T_{k}+T_{k-2}
\end{aligned}
$$


and prove that $L>0$. We distinguish two cases:

A) For $\alpha_{k}=0$, we have:

$$
L=\sum_{m=2}^{k-3}\left(2-\alpha_{m}\right) T_{m}-\alpha_{1}-\alpha_{k-1} T_{k-1}+T_{k-2}+\left(2-\alpha_{k-2}\right) T_{k-2}
$$

then:

$$
L \geq \sum_{m=2}^{k-3}\left(2-\alpha_{m}\right) T_{m}+2 T_{k-2}-T_{k-1}-1
$$

Since:

$$
\sum_{m=2}^{k-3}\left(2-\alpha_{m}\right) T_{m}>0, k \geq 6
$$

and from above:

$$
2 T_{k-2}-T_{k-1}-1 \geq 0
$$

Consequently $L>0$.

B) $\alpha_{k}=1$. Which implies in this case that $\alpha_{k-1}=\alpha_{k-2}=$ 0. So:

$$
\begin{aligned}
& L=\sum_{m=2}^{k-2}\left(2-\alpha_{m}\right) T_{m}-\alpha_{1}-T_{k}+T_{k-2} \\
& =\sum_{m=2}^{k-3}\left(2-\alpha_{m}\right) T_{m}+\left(2-\alpha_{k-2}\right) T_{k-2}-\alpha_{1}-T_{k}+T_{k-2} \\
& =\sum_{m=2}^{k-3}\left(2-\alpha_{m}\right) T_{m}+3 T_{k-2}-T_{k}-\alpha_{1} \\
& =\sum_{m=2}^{k-4}\left(2-\alpha_{m}\right) T_{m}+\left(2-\alpha_{k-3}\right) T_{k-3}+3 T_{k-2}-T_{k}-\alpha_{1} \\
& \geq \sum_{m=2}^{k-4}\left(2-\alpha_{m}\right) T_{m}+T_{k-3}+3 T_{k-2}-T_{k}-1
\end{aligned}
$$

From:

$$
T_{k}=T_{k-1}+T_{k-2}+T_{k-3}
$$

we get:

$$
L \geq \sum_{m=2}^{k-4}\left(2-\alpha_{m}\right) T_{m}+2 T_{k-2}-T_{k-1}-1
$$

Since:

$$
2 T_{k-2}-T_{k-1}-1 \geq 0
$$

and:

$$
\sum_{m=2}^{k-4}\left(2-\alpha_{m}\right) T_{m}>0
$$

then:

$$
L>0
$$

Consequently:

$$
\sum_{m=1}^{k} \alpha_{m} T_{m}<2 \sum_{m=2}^{k-2} T_{m}+T_{k-2}, \text { for every } k \geq 6
$$

Which completes the proof of lemma.

Let us return to the proof of theorem 2.2 by taking $N$ $\geq 1$ and assuming that $\mathrm{N}$ has two non identical representations

$$
N=\sum_{m=1}^{\infty} \alpha_{m} T_{m}=\sum_{m=1}^{\infty} \beta_{m} T_{m}
$$

where:

$$
\left\{\begin{array}{l}
\alpha_{m} \alpha_{m+1}=0 \\
\alpha_{m} \alpha_{m+2}=0
\end{array}, \text { for } m \geq 1\right.
$$

and the same constraints for $\beta_{m}(m \geq 1)$.

That is, $\sum_{1}^{\infty}\left|\alpha_{m}-\beta_{m}\right| \neq 0$. Let $k$ be the largest value of $m$ such that $\alpha_{k} \neq \beta_{k}$, we may assume without loss of generality that $\alpha_{k} \neq 1$ and $\beta_{k}=0$. Since the validity of lemma 2.1 and proposition 2.1 is for $k \geq 6$, we distinguish in the sequel two cases.

A) $1 \leq k \leq 6$.

$k=1$

This means that $\alpha_{1}=1, \beta_{1}=0$ and $\alpha_{m}=\beta_{m}$, for $m \geq 2$. Since:

$$
\sum_{m=2}^{+\infty} \alpha_{m} T_{m}=\sum_{m=2}^{+\infty} \beta_{m} T_{m}
$$

and:

$$
\begin{aligned}
& \sum_{m=1}^{1} \alpha_{m} T_{m}+\sum_{m=2}^{+\infty} \alpha_{m} T_{m}=1+\sum_{m=2}^{+\infty} \alpha_{m} T_{m} \neq \sum_{m=1}^{+\infty} \beta_{m} T_{m} \\
& +\sum_{m=2}^{+\infty} \beta_{m} T_{m}=0+\sum_{m=2}^{+\infty} \beta_{m} T_{m}
\end{aligned}
$$

there is not two non identical representations, because $N$ cannot be represented by two different values.

$k=2$

Which yields $\alpha_{2}=1, \beta_{2}=0$ and $\alpha_{m}=\beta_{m}$ for $m \geq 3$.

With these coefficients we can represent $N$ as: 


$$
N=\sum_{m=1}^{+\infty} \alpha_{m} T_{m}=\sum_{m=1}^{+\infty} \beta_{m} T_{m}
$$

if and only if $\alpha_{1}=0$ and $\beta_{1}=1$. Thus:

$$
N=0 . T_{1}+1 . T_{2}+\sum_{m=3}^{+\infty} \alpha_{m} T_{m}=1 . T_{1}+0 . T_{2}+\sum_{m=3}^{+\infty} \beta_{m} T_{m}
$$

but a such representations are identical in the sense of the definition 1.1. If $\beta_{1}=0$, then:

$$
\sum_{m=1}^{+\infty} \alpha_{m} T_{m} \neq \sum_{m=1}^{+\infty} \beta_{m} T_{m}
$$

and there are not two non identical representations, since $N$ can not be represented by two different values.

$k=3$.

That is, $\alpha_{3}=1, \beta_{3}=0$ and for $m \geq 4, \alpha_{m}=\beta_{m}$. In this case the greatest value of $\sum_{m=1}^{3} \beta_{m} T_{m}$ is 1 and in contrast $\sum_{m=1}^{3} \alpha_{m} T_{m}=2$. Hence:

$$
\sum_{m=1}^{3} \alpha_{m} T_{m}+\sum_{m=4}^{+\infty} \alpha_{m} T_{m}=2+\sum_{m=4}^{+\infty} \alpha_{m} T_{m} \neq \sum_{m=1}^{3} \beta_{m} T_{m}+\sum_{m=4}^{+\infty} \beta_{m} T_{m}
$$

Then, as above, there are not two non identical representations, since $N$ can not be represented by two different values.

$k=4$

That is, $\alpha_{4}=1, \beta_{4}=0$ and for $m \geq 5, \alpha_{m}=\beta_{m}$. In this case the greatest value of $\sum_{m=1}^{4} \beta_{m} T_{m}$ is 2 and in contrast the smallest value of $\sum_{m=1}^{4} \alpha_{m} T_{m}$ is 4 . Hence, $N$ can not be represented by two different values, that is, there is not two non identical representations.

$k=5$

That is, $\alpha_{5}=1, \beta_{5}=0$ and for $m \geq 6, \alpha_{m}=\beta_{m}$. In this case the greatest value of $\sum_{m=1}^{5} \beta_{m} T_{m}$ is 5 and in contrast the smallest value of $\sum_{m=1}^{5} \alpha_{m} T_{m}$ is 7 . Thus, as above there is not two non identical representations.

$k=6$

That is, $\alpha_{6}=1, \beta_{6}=0$ and for $m \geq 7, \alpha_{m}=\beta_{m}$. In this case the greatest value of $\sum_{m=1}^{6} \beta_{m} T_{m}$ is 8 and in contrast the smallest value of $\sum_{m=1}^{6} \alpha_{m} T_{m}$ is 13 . Thus, as in the previous cases $N$ can not be represented by two different values, that is, there is not two non identical representations.

B) $7 \leq k$. From the fact that $\alpha_{m}=\beta_{m}$ for $m \geq k+1$ and:

$$
N=\sum_{m=1}^{+\infty} \alpha_{m} T_{m}=\sum_{m=1}^{+\infty} \beta_{m} T_{m}
$$

we have:

$$
\sum_{m=1}^{k} \alpha_{m} T_{m}=\sum_{m=1}^{k} \beta_{m} T_{m}
$$

Putting:

$$
l=\sum_{m=1}^{k} \alpha_{m} T_{m}=\sum_{m=1}^{k} \beta_{m} T_{m}
$$

Then:

$$
l=\sum_{m=1}^{k} \alpha_{m} T_{m}=\sum_{m=1}^{k-1} \beta_{m} T_{m}
$$

since $\beta_{k}=0$. On the other hand $l \leq N$ and we can write:

$$
l=\sum_{m=1}^{k} \alpha_{m} T_{m}=\sum_{m=1}^{k-1} \alpha_{m} T_{m}+T_{k} \geq T_{k}
$$

According to lemma 2.1. we have:

$$
l=\sum_{m=1}^{k-1} \beta_{m} T_{m}<2 \sum_{m=2}^{k-3} T_{m}+T_{k-3}
$$

And by proposition 2.1, we have:

$$
T_{k}-3-T_{k-2}=2 \sum_{m=2}^{k-3} T_{m}
$$

So:

$$
l<T_{k}-3-T_{k-2}+T_{k-3}
$$

By the recurrent relation of $T_{n}$, we have:

$$
l<T_{k-1}+T_{k-2}+T_{k-3}-3-T_{k-2}+T_{k-3}
$$

That is:

$$
l<T_{k-1}-3+2 T_{k-3}
$$

Now to get $l<T_{k}$, we will prove that:

$$
T_{k-1}-3+2 T_{k-3}<T_{k}
$$

Indeed: 


$$
\begin{aligned}
& T_{k}-T_{k-1}+3-2 T_{k-3}=T_{k-1}+T_{k-2}+T_{k-3}-T_{k-1}+3-2 T_{k-3} \\
& =T_{k-2}+3-T_{k-3} \\
& =T_{k-3}+T_{k-4}+T_{k-5}+3-T_{k-3} \\
& =T_{k-4}+T_{k-5}+3>0
\end{aligned}
$$

Then:

$$
l<T_{k-1}-3+2 T_{k-3}<T_{k}
$$

Finally, we have:

$$
T_{k} \leq l<T_{k}
$$

which is a contradiction. Hence the representation is unique.

\section{Applications and Perspectives}

The representation of a positive integer $\mathrm{n}$ by a sum of elements of a given sequence is an interesting problem which is well known in the mathematical literature; namely, unique representation of integers as sum of distinct Lucas numbers (Brown, Jr, 1969), Fibonacci and Lucas representation (Pihko, 1986), Cantor's development of a positive integer (Mercier, 2004),... . Our problem set in this context but with a well addition which is the uniqueness of representation.

As a perspective, the techniques used in our work can be employed in other problems for the same purposes. In this sense we can consider, for example, the case of the generalized tribonacci sequences and higher orders (Pentanacci, hexanacci, ...k-Fibonacci sequence..).

This field of mathematics which focuses on the study of words and formal languages combinatorics on words affects various areas of mathematics study, including algebra and computer science. Combinatorics of words is connected to many modern, as well as classical, fields of mathematics. Connections to combinatorics-actually being part of it - are obvious, but also connections to algebra are deep. Indeed, a natural environment of a word is a free semigroup.

More generally, the above connections can be illustrated as in Fig. 1 (Karhumaki, J.).

For more clarification we can take the sequences of words like the Fibonacci sequence of words on the binary alphabet $\{0,1\}$ can be de.ned by the recurrent relation:

$$
\left\{\begin{array}{c}
F_{1}=1, F_{2}=0 \\
F_{n}=F_{n-1} F_{n-2}(\text { with theconcatenation product })
\end{array}\right.
$$

$F_{1}=1, F_{2}=0, F_{3}=01, F_{4}=010, \ldots$. For further references on the subject see for example (Lotaire, 2002; Karhumaki and Berstel, 2003).

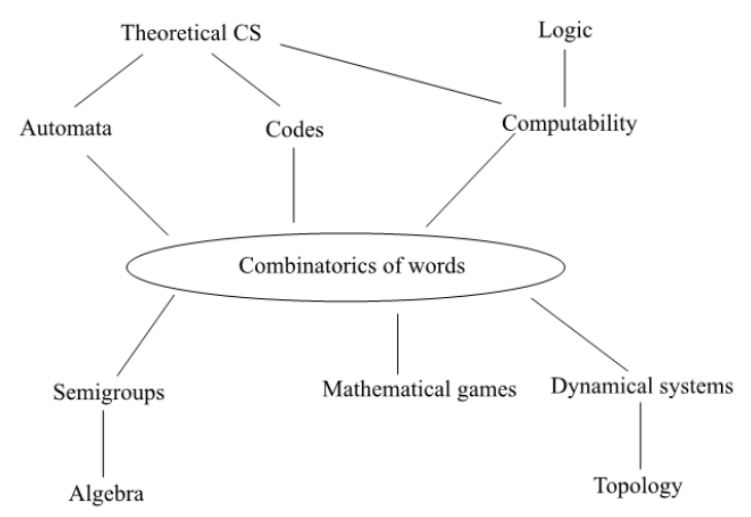

Fig. 1. Reference grid of the relationship between the fields of applications

\section{Acknowledgment}

The authors would like to thank an anonymous referee for reading this article and the suggestions given.

\section{Author's Contributions}

The authors worked together during the preparation of this article.

\section{Ethics}

The present work is really ours and there will be no ethical problem.

\section{References}

Brown, Jr, J.L., 1969. Unique representation of integers as sum of distinct Lucas numbers. The Pennsylvania State University.

Karhumaki, J. and J. Berstel 2003. Combinatorics of words. Tutorial Bull EATCS, 79: 179-228.

Karhumaki, J. Combinatorics of words. https:/www.utu.fi/en/units/sci/units/math/staff/Doc uments/karhumaki/combwo.pdf

Koshy, T., 2001. Fibonacci and Lucas Numbers with Applications. 1st End., Wiley, New York, ISBN-10: 0471399698, pp: 672.

Lotaire, M., 2002. Algebraic Combinatorics on Words. 1st Edn., Combridge University Press, Cambridge, ISBN-10: 0521812208, pp: 504.

Mercier, A., 2004. 1001 problèmes en théorie des nombres, ellipses.

Pihko, J., 1986. On Fibonacci and Lucas representation and theorem of Lekkerkerker. University of Helsinki, Hallituskatu 15 SF-00100 Helsinki, Finland. 